\title{
Solar Load Ratio and ISO 13790 methodologies: indirect gains from sunspaces
}

\author{
Marta J. N. Oliveira Panão, Susana M.L. Camelo and Helder J. P. \\ Gonçalves, \\ National Laboratory of Energy and Geology (LNEG), Estrada do Paço Lumiar, 22, \\ 1649-038 Lisbon, Portugal
}

\begin{abstract}
This paper reviews and analyzes the compatibility of the simplified empirical method based on the dimensionless parameter of Solar Load Ratio (SLR) and the monthly procedure of the standard ISO 13790 for indirect gains, specifically for unconditioned zones adjacent to a conditioned zone, but separated from it by a partition wall (sunspaces).

The main contribution of the work presented here is the new formulation to account SLR correlations in ISO 13790, obtained for sunspaces, but generalizable for other solar systems with known SLR functions.

Simulation models are used to perform a sensitivity analysis of internal gains and heat transfer through solar collector surfaces, both issues that distinguishes ISO 13790 from Load Ratio methods. The analysis shows that internal gains can be added to the heat source term or subtracted to heat transfer term without influencing the utilization factor dependence with the gain-to-loss ratio. On the other hand, the SLR assumption that solar collector surfaces are neutral elements and, therefore, not added to the heat transfer term, results in large inconsistencies between SLR and ISO 13790 methods.

The detailed monthly methodology of ISO 13790 fairly reproduces results obtained by simulation. However, predictions from the simplified monthly methodology of ISO 13790 fail in mid season months and coldest months.

Keywords: solar indirect gains, sunspaces, ISO 13790, Solar Load Ratio, utilization factor
\end{abstract}

\section{Introduction}

The integration of solar passive systems in buildings is of utmost importance to decrease thermal energy demand, especially for residential use. The 
EPBD Recast, Directive 2010/31/EU [1], sets that each European Member State should regulate in order to achieve, for new buildings in 2020, nearly Zero Energy Buildings (nZEB, also known as Net Zero Energy Buildings). Although there is not a common definition among all countries [2], it is conceptually accepted as necessary to reduce the energy demand for heating and ventilation and, for warmer climates, also for cooling. Moreover, the remaining energy demand, or at least a substantial part of it, should be covered by energy produced using renewable sources in the building itself or nearby.

The European Energy Labeling, imposed in most European Member States, pursuant to the EPBD Directive 2002/91/EC [3], aims at harmonizing the energy calculations based on standard ISO 13790 [4]. The standard includes two calculation procedures for heating and cooling energy needs: 1) an hourly energy balance of a single zone where transient effects are simplified by a capacitance equivalence approach and 2) an energy balance for a longer period, typically one month or one season, assuming quasi-steady state conditions. The procedure for the inclusion of solar systems, such as unconditioned zones, opaque elements with transparent insulation, ventilated solar walls (Trombe walls) and ventilated envelope elements, is fully described in Annex E of the standard.

The increase of scientific knowledge and computation capabilities tend to intensify the calculation complexity of building thermal regulations. On the other hand, a few decades ago, where computational resources were scarce, it is possible to find multiple handling methods to compute solar systems contribution $[5,6,7,8]$. Moreover, regulation procedures should also be easily handled by building professionals in the building sector. Therefore, in this sense, this paper reviews and analyzes the integration of the simplified empirical method SLR [5], based on the dimensionless parameter of Solar Load Ratio, in ISO 13790 for sunspaces, which are appropriate and effective solar systems during the cold period of the year $[9,10]$. Due to the fact that sunspaces are passive heating systems, this study focuses only on the heating season, despite the cooling season is also included in ISO 13790.

\section{Heating energy needs}

In ISO 13790, heating energy needs are calculated by a quasi-steady state method applied to a defined period, from one month to the entire heating season. But, in order to make SLR and ISO 13790 methods comparable and due to the fact that SLR is applied on a monthly basis, the monthly period analysis is also adopted for the ISO 13790 approach. For SLR method, heating energy needs are empirically calculated taking into account solar gains and heating energy demand considering no solar gains, identified as 
'reference heating load'. Therefore, this section reviews the monthly method of ISO 13790 for heating energy needs calculation and explores how indirect gains from unconditioned zones are included. It reviews also SLR method and evaluates the integrability of both methods.

\subsection{ISO 13790: Base method}

The method developed by Dijk et al. [11], also described in detail in ISO 13790 [4], consists of a numerical estimative of the physical quantities of the monthly heat transfer $\left(Q_{h t}\right)$ and heat sources $\left(Q_{g n}\right)$, which differs from a mere comparison between gains and losses. The energy balance is applied to a small building, typically a single family house or an apartment, simplified into a single thermal zone. Other adjacent conditioned zones are treated as similar and surfaces between them are adiabatic. Adjacent unconditioned zones with lower average air temperature are considered by the inclusion of an adjustment factor which corrects the inside-to-outside air temperature difference. The heat transferred by ventilation (including infiltration) and transmission (conduction, convection and longwave radiation exchange with surroundings) directly depends on the air temperature difference between inside and outside and, therefore, is part of the first term. The exchange of energy which does not fit in the first term constitutes the heat sources, e.g. shortwave radiative gains, extra longwave radiation exchange with sky vault and internal gains. The calculation of heating energy needs, given by

$$
Q_{n d}=Q_{h t}-\eta_{g n} Q_{g n}
$$

uses the gain utilization factor $-\eta_{g n}$ - which is approximated by the expression

$$
\eta_{g n}=\frac{1-\gamma^{a}}{1-\gamma^{a+1}}
$$

where $\gamma$ is the heat sources-to-heat transfer ratio, $\gamma=Q_{g n} / Q_{h t}$. In equation (2), $a$ is an adjustable parameter depending on the building time constant $(\tau)$ and, therefore, on its thermal inertia. Equation (2) is valid only for $\gamma>0$ and $\gamma \neq 1$; therefore, for $\gamma=1, \eta_{g n}=a /(a+1)$ and for $\gamma \leq 0, \eta_{g n}=0$.

\subsection{ISO 13790: Indirect gains}

Indirect gains are those collected in an adjacent, but unconditioned zone, such as sunspaces (conservatories), atriums or massive solar walls. For sunspaces - specifically those which are adjacent to a conditioned zone but separated from it by a partition wall, - the glazed surfaces collect heat from the sun which is stored in sunspaces massive surfaces. Part of the heat is transferred to the conditioned zone by transmission through the partition surfaces 
- either opaque or transparent - and by the exchange of air between zones. Moreover, when a transparent surface (window) separates the conditioned zone from the sunspace, part of the solar radiation is directly transmitted to the conditioned zone $\left(Q_{s d, t}\right)$. ISO 13790 also accounts for the part of solar radiation directly absorbed by the opaque partition wall $\left(Q_{s d, p}\right)$. The remaining heat absorbed by other sunspace massive surfaces $\left(Q_{a b, s}\right)$ is, later on, transferred to the external environment through the sunspace external surfaces or indirectly transferred to the conditioned zone (see Fig. 1). The sunspace adjustment factor, $b_{t r}$, which is a characteristic of the sunspace, defines the parcel of heat that is transferred to the outside.

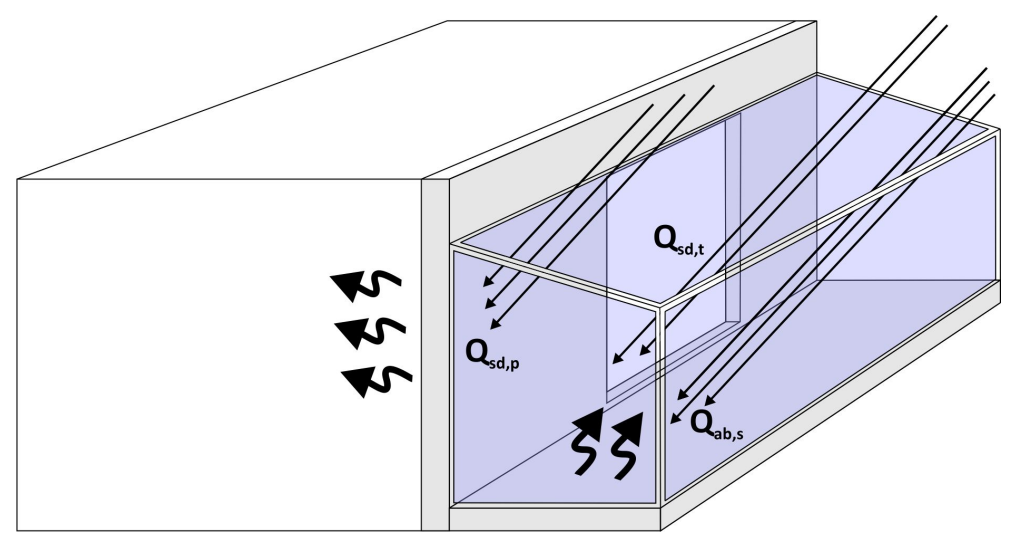

Figure 1: Sunspace direct and indirect gains.

For each opaque sunspace surface $j$, solar gains at the sunspace are defined by the sum of the product of the monthly solar irradiation in each surface $\left(I_{j}\right)$, its solar absorption coefficient $\left(\alpha_{j}\right)$ and the effective collection area $\left(A_{e j}\right)$.

$$
Q_{a b, s}=\sum_{j} \alpha_{j} I_{j} A_{e j}
$$

where $A_{e j}$ takes into account the reduction coefficients corresponding to sunspace shading elements, window frame and glazing total solar transmission.

Indirect gains are calculated by summing the solar heat gains absorbed by sunspace surfaces, but deducting the direct gains through the opaque partition wall and released to the conditioned zone.

$$
Q_{s i}=Q_{a b, s}\left(1-b_{t r}\right)-Q_{s d, p}
$$

Therefore, the total amount of heat transferred from the sunspace to the conditioned zone $\left(Q_{s s}\right)$ is given by the direct and indirect parcels 


$$
Q_{s s}=Q_{s d, t}+Q_{s d, p}+Q_{s i}
$$

which, taking in consideration equation (4), reduces to

$$
Q_{s s}=Q_{s d, t}+Q_{a b, s}\left(1-b_{t r}\right)
$$

Henceforth in this text, unlike ISO 13790, sunspace indirect gains also include the component $Q_{s d, p}$, therefore indirect gains are here identified by the second term of equation (6).

Alternatively to the described method - hereafter identified by detailed method - a simplified method is proposed in ISO 13790, based on a correction of the adjustment factor, $b_{t r}^{*}$, in order to include sunspace solar gains during heating or cooling seasons. For the warmer months, the corrected adjustment factor could even take negative values. The calculation of $b_{t r}^{*}$ follows ISO 13789 [12], where

$$
b_{t r}^{*}=b_{t r}-\frac{\Phi_{u}}{\left(H_{p}+H_{s}\right)\left(T_{r e f}-T_{e x t}\right)}
$$

with $H_{p}$ as the sunspace partition wall conductance (conditioned-to-unconditioned zone conductance), $H_{s}$ as the sunspace external surfaces conductance (unconditioned zone-to-external conductance) and, by definition, $\Phi_{u}$ as the heat generated within the unconditioned zone; however ISO 13789 does not present a formulation to account for this last term. $T_{r e f}$ and $T_{\text {ext }}$ are the reference value assumed for heating set point and the monthly average of external air temperature, respectively.

The corrected adjustment factor is used for the calculation of the corrected heat transfer through the sunspace partition wall, as

$$
Q_{h t, p}^{*}=H_{p}\left(T_{r e f}-T_{e x t}\right) b_{t r}^{*}
$$

From equations (7) and (8), it can be shown that sunspace gains are included as a subtracting term in the heat transfer corresponding to the partition wall of the unconditioned zone $\left(Q_{h t, p}\right)$ and the corrected heat transfer through the sunspace partition wall is given by

$$
Q_{h t, p}^{*}=Q_{h t, p}-\Phi_{u}\left(1-b_{t r}\right)
$$

It is noteworthy that $Q_{h t, p}$ is already accounted for in the detailed methodology and included in the general term $Q_{h t}$.

In synthesis (see Table 1), the detailed method consists of adding the direct - $Q_{s d, t}$ - and indirect solar heat gains from the sunspace - $Q_{a b, s}\left(1-b_{t r}\right)$ - to the base case heat source, i.e. conditioned room direct gains - $Q_{s d, w}$ - and 
internal gains $-Q_{i}$. Otherwise, the simplified method consists of subtracting to the conditioned zone heat transfer, $Q_{h t}$, the indirect solar heat gains from the sunspace, $\Phi_{u}\left(1-b_{t r}\right)$. In the simplified method, direct solar heat gains from window partitions - $Q_{s d, t}$ - are still considered as heat sources.

Table 1: ISO 13790: Heat transfer and heat sources terms by method.

\begin{tabular}{lll}
\hline method & heat transfer & heat source \\
\hline base case & $Q_{h t}$ & $Q_{s d, w}+Q_{i}$ \\
detailed method & $Q_{h t}$ & $Q_{s d, w}+Q_{i}+Q_{s d, t}+Q_{a b, s}\left(1-b_{t r}\right)$ \\
simplified method & $Q_{h t}-\Phi_{u}\left(1-b_{t r}\right)$ & $Q_{s d, w}+Q_{i}+Q_{s d, t}$ \\
\hline
\end{tabular}

It is noteworthy that $Q_{h t}$ in Table 1 includes all the surfaces that form the thermal envelope of the conditioned zone, sunspace partition walls included.

\subsection{Load Ratio methods}

During the 80's, the SLR (Solar Load Ratio) method [5, 13], developed by the Los Alamos Scientific Laboratory, was widely used to quantify monthly heating energy needs of passive solar houses with direct and indirect gains. This method consists in comparing the collected solar gains $\left(Q_{\text {sol }}\right)$ with its reference heating load $\left(Q_{r e f}\right)$, which is the energy that the building would require if there were no solar gains; the ratio between them is the SLR parameter. It is noteworthy that the 'reference heating load' is an energy demand, instead of a heat load as it is commonly defined. Theoretically, for a building with an infinite thermal capacity, when monthly solar gains are higher than the reference heating load ( $\mathrm{SLR}>1$ ), heating energy would not be required. But, for real buildings, part of the total solar gains are not useful because the building storage capacity is limited, so that extra solar gains cause an increase of the sensible air temperature above the reference set point air temperature. Therefore, the Solar Heating Fraction (SHF), also named Solar Saving Fraction [14], quantifies the parcel of solar gains that are useful to the reference heating load. In SLR method, SHF is obtained by empirical correlations (e.g. [14]) and heating energy needs are calculated from

$$
Q_{n d}=(1-\mathrm{SHF}) Q_{r e f}
$$

SHF correlation coefficients are obtained by fitting different mathematical functions to the known results obtained by running experiments or simulations. It is noteworthy that correlation functions are strongly dependent from the predefined conditions, such as orientation, building materials, insulation, glazing type, ventilation, etc. 
Subsequently, numerous other methods are developed, which differ from SLR more in details than in its essence. For example, the Gain Load Ratio (GLR) method [8], as ISO 13790, for the reference heating load calculation takes into account the heat transferred through the solar collector surface window, storage wall, sunspace partition wall - and solar gains are accounted for other orientations besides south, but SLR does not. It is noteworthy that both methods, SLR and GLR, consider that internal gains are subtracted from the reference heating load and, therefore, are not included in the heat source term as in ISO 13790.

For the specific case of the sunspaces [14], SHF is a function of a corrected Solar Load Ratio, SLR*, equivalent also to a gain-to-loss ratio, where the gain parcel is given by the solar radiation absorbed in the sunspace surfaces, here identified as $Q_{a b, s}$, minus the effective sunspace heat transfer, which is obtained by $Q_{h t, s}$ corrected by an empiric parameter, $\xi$,

$$
\mathrm{SLR}^{*}=\frac{Q_{a b, s}-\xi Q_{h t, s}}{Q_{r e f}}
$$

where $Q_{\text {ref }}$ is still the reference heating load of the conditioned zone.

In the literature on the SLR method applied to sunspaces, a physical meaning is not given to $\xi$, despite its relation with sunspace heat transfer 'effectiveness'. However, it can be verified from [14] that it decreases with the use of night insulation at sunspace glazing and the use of masonry instead of an insulated partition wall, supporting the fact that it is related with the sunspace thermal storage.

The validity of the correlations presented in [14] for predefined geometries, with $b$ and $c$ as empirical parameters, given by a general expression of

$$
\mathrm{SHF}=1-b \exp \left(-c \times \mathrm{SLR}^{*}\right)
$$

holds on the assumptions that only gains from sunspace are considered for the conditioned zone (no additional zone gains such as direct or internal gains are considered) and sunspace partition wall is totally opaque.

The solar contribution of multiple solar systems, $\mathrm{SHF}^{M}$, such as the combination of south windows with sunspaces, can be estimated by the SHF of each system $i\left(\mathrm{SHF}_{i}\right)$ weighted by their glazing projected area $\left(A_{p_{i}}\right)$. The procedure also assumes that each system is independent of other systems, with a projected area corresponding to the sum of all.

$$
\mathrm{SHF}^{M}=\sum_{i} a_{p_{i}} \mathrm{SHF}_{i}
$$

with $a_{p_{i}}$ as the fraction of the projected area $\left(A_{p_{i}}\right)$ from the total $\left(\sum_{i} A_{p_{i}}\right)$. 
For vertical south windows, $A_{p}$ is just the net glazing area (no frame included) and for sunspaces it is also the net glazing area, but projected on a vertical plane.

\subsection{Load Ratio methods and ISO 13790}

Similarities among Load Ratio methods and ISO 13790 are found in Sander and Barakat [8] and Oliveira and Oliveira Fernandes [15] when the utilization factor concept is introduced, referred to utilization factor for solar gains and utilization efficiency of solar gains, respectively. In [8], the utilization factor is a function of thermal mass and gain-to-loss ratio and is correlated with GLR by plots similar to those adopted in ISO 13790.

On the other hand, in [15], the utilization efficiency of solar gains is a function of the building time constant, $\tau$ (in hours) and the non-utilizability for the zero thermal inertia, $\phi$, which is the monthly fraction of solar gains that are not useful considering any heat storage. The dependence among parameters is expressed by

$$
\mathrm{SHF}=\eta_{g n}(\phi, \tau) \mathrm{SLR}
$$

From equations (10) and (14), the following expression is derived

$$
Q_{n d}=\left(1-\eta_{g n} S L R\right) Q_{r e f}
$$

Considering that SLR expresses a gain-to-loss ratio, equation (15) is similar to equation (1) used in ISO 13790 for heating energy needs determination. Moreover, comparing (10) with the alternative formulation of ISO 13790

$$
Q_{n d}=\left(1-\eta_{l s}\right) Q_{h t}
$$

it can be concluded that SHF is equivalent to the loss utilization factor, $\eta_{l s}$, calculated by

$$
\eta_{l s}=\frac{1-(1 / \gamma)^{a}}{1-(1 / \gamma)^{a+1}}
$$

It is noteworthy that $\gamma$ and SLR are both gain-to-loss ratios, even if the methodology of calculation is different as synthesized in Table 2 .

In Table 2, $Q_{h t, p}$ refers to the heat transfer through the solar collector surfaces, which contributes to solar gains, e.g. window, sunspace partition walls, storage walls, etc. 
Table 2: Heat source-to-heat transfer (gain-to-loss) ratio with indirect gains. No transparent sunspace partition wall.

\begin{tabular}{ll|llll}
\hline method & $\begin{array}{l}\text { gain- } \\
\text { to-loss } \\
\text { ratio }\end{array}$ & $\begin{array}{l}\text { heat } \\
\text { fer/loss }\end{array}$ & trans- & $\begin{array}{l}\text { heat } \\
\text { sources/gain }\end{array}$ & $\begin{array}{l}\text { indirect gains } \\
\left(Q_{s s}\right)\end{array}$ \\
\hline $\begin{array}{l}\text { base case } \\
\text { detailed ISO }\end{array}$ & $\gamma$ & $Q_{h t}$ & $Q_{s d, w}+Q_{i}$ & \\
$\begin{array}{l}13790 \\
\text { simplified ISO }\end{array}$ & $\gamma$ & $Q_{h t}$ & $Q_{s d, w}+Q_{i}+Q_{s s}$ & $Q_{a b, s}\left(1-b_{t r}\right)$ \\
$\begin{array}{l}13790 \\
\text { SLR method }\end{array}$ & SLR & $Q_{h t}-Q_{s s}$ & $Q_{s d, w}+Q_{i}$ & $\Phi_{u}\left(1-b_{t r}\right)$ \\
\hline
\end{tabular}

\subsection{Research aim}

From the comparison of methodologies, there are a number of questions that should be considered in order to take into account indirect gains from passive solar systems. Are internal/indirect gains the same as reducing the heat transfer term instead of being added to the heat source term? How to quantify indirect gains? Could the gain utilization expressions $\left(\eta_{g n}\right)$ be globally applied for any passive solar system? How to integrate known SLR correlations into the ISO 13790 methodology? How to account for the solar contribution of multiple passive solar systems in ISO 13790?

The goal of this paper is, therefore, twofold: i) evaluate the performance of the indirect gains methodology of ISO 13790 and ii) study how to integrate SLR correlations into the methodology of ISO 13790 in order to give continuity of the correlations of Solar Passive Handbooks [14].

To that end different geometries of sunspaces are evaluated, but for the sake of simplicity, the sunspace partition wall is totally opaque (no direct transmitted gains are considered, $Q_{s d, t}=0$ ). Furthermore, in order to minimize the errors associated with the physical parameters determination, whenever possible, the global terms - such as heat transfer, heat sources, adjustment factor - are directly obtained by simulation (see Section 3.2).

\section{Sunspaces}

\subsection{Case-studies description}

The case study is a single-family house located in Lisbon $\left(38.8^{\circ} \mathrm{N}, 9.1^{\circ} \mathrm{W}\right)$ with $63 \mathrm{~m}^{2}$ of net floor area. Windows - direct gain systems - are all located in the south façade and are double glazed with total solar transmission of 0.69. There are two types of tested sunspaces according to its position to the building block: (A) attached and (B) integrated (Figs. 2 and 3, respectively). 
Glazing surfaces of sunspaces have a total solar transmission of 0.81 and differ in tilt angle according to their type (see Table 3).

Table 3: Case studies with and without sunspaces.

\begin{tabular}{|c|c|c|c|c|c|}
\hline \multirow{3}{*}{$\begin{array}{l}\text { model } \\
\text { type }\end{array}$} & \multirow[b]{3}{*}{$A_{s d, w} / A_{p}[\%]$} & \multicolumn{3}{|c|}{ Sunspaces glazing (Ind.Gains) } & \multirow{3}{*}{$\begin{array}{c}\text { South glazing (Dir.Gains) } \\
\text { vertical } \\
\text { area }\left[\mathrm{m}^{2}\right]\end{array}$} \\
\hline & & vertical & & lit & \\
\hline & & area $\left[m^{2}\right]$ & area $\left[m^{2}\right]$ & angle $[$ deg] & \\
\hline A1 & 0 & 11.8 & 11.3 & 30 & - \\
\hline A1 & 30 & 8.3 & 7.9 & 30 & 4.0 \\
\hline A1 & 70 & 3.5 & 3.3 & 30 & 10.6 \\
\hline $\mathrm{A} 2$ & 0 & - & 23.4 & 50 & - \\
\hline $\mathrm{A} 2$ & 30 & - & 16.4 & 50 & 4.0 \\
\hline $\mathrm{A} 2$ & 70 & - & 6.9 & 50 & 10.6 \\
\hline A3 & 100 & - & - & - & 15.5 \\
\hline B1 & 0 & 10.7 & 10.7 & 30 & - \\
\hline B1 & 50 & 10.7 & 10.7 & 30 & 9.7 \\
\hline B2 & 0 & - & 17.4 & 50 & - \\
\hline B2 & 50 & - & 17.4 & 50 & 9.7 \\
\hline B3 & 50 & - & - & - & 9.7 \\
\hline
\end{tabular}

A attached, B integrated

\subsection{Assessment of heat transfer and heat sources terms by simulation}

The seasonal quasi steady-state method described in Section 2.1 uses a heat transfer/heat sources approach. Therefore, instead of a gain/loss energy balance which is generally the output available in the software tools, heat transfer and heat sources are calculated separately using the methodology described in Corrado and Fabrizio [16], and also used in Oliveira Panão et al. [17]. The procedure consists of running three simulations, using the building energy simulation program EnergyPlus [18], for each model or parametric variation described by:

1. Canceling all heat sources - solar gains, sky vault extra longwave radiative exchanges and internal gains - and using both ideal heating and cooling systems with the setpoint temperatures at $293 K$, the reference temperature for winter $\left(20^{\circ} \mathrm{C}\right)$, in order to calculate the heat transfer term from the heating $\left(Q_{H, 20}^{(1)}\right)$ and cooling energy needs $\left(Q_{C, 20}^{(1)}\right)$, by

$$
Q_{h t}=Q_{H, 20}^{(1)}-Q_{C, 20}^{(1)}
$$




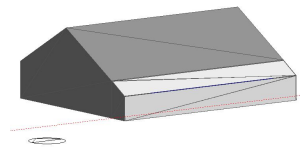

(a) $0 \%$ Dir.Gains

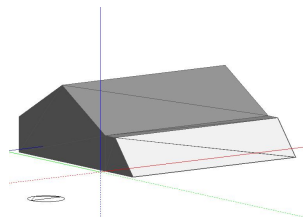

(e) $0 \%$ Dir.Gains

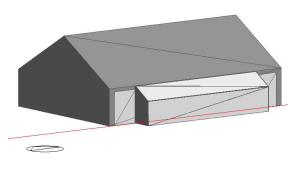

(b) $30 \%$ Dir.Gains

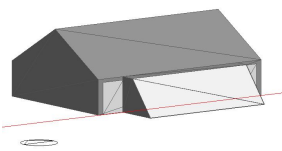

(f) $30 \%$ Dir.Gains

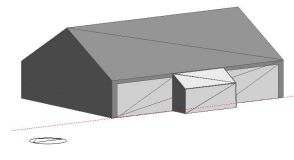

(c) $70 \%$ Dir.Gains

(d) $100 \%$ Dir.Gains
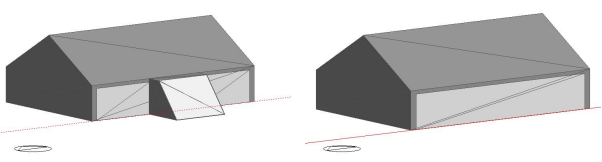

(g) $70 \%$ Dir.Gains (h) $100 \%$ Dir.Gains

Figure 2: Attached sunspace: A1 (a, b and c), A2 (e, f and g). No sunspace: A0 (d and h).

2. Including all heat sources and repeating simulations at the conditions defined previously to get $Q_{H, 20}^{(2)}$ and $Q_{C, 20}^{(2)}$ and, afterwards, calculate the heat sources term by

$$
Q_{g n}=Q_{h t}-\left(Q_{H, 20}^{(2)}-Q_{C, 20}^{(2)}\right)
$$

3. Run a last simulation to obtain heating energy needs,

$$
Q_{n d}=Q_{H, 20}^{(3)}
$$

with an ideal system with the setpoint temperature at $293 K$.

The simulation condition, ensuring no solar gains, consists of using hourly climate data where solar radiation is nulled. In order to consider that building surfaces are totally enclosed, the sky longwave downward radiative exchange is assumed as equal to the emitted by a surface at the external temperature and emissivity of 0.9 , which is verified for most of the building materials. It is noteworthy that cooling energy needs in equations (18) to (19) are always assumed to be positive.

For calculation of the corrected heat transfer through the sunspace partition wall, with equation (9), it is necessary to achieve by simulation monthly values for $\Phi_{u}$ and $b_{t r}$. For this, a fourth simulation is run which is similar to the first one, but replacing the partition walls by adiabatic surfaces and using a set point air temperature at $293 K$ (heating and cooling).

This simulation is run for both conditioned and sunspace zones, and the obtained results are $Q_{H, 20}^{(4)}$ and $Q_{C, 20}^{(4)}$ for the conditioned zone and $Q_{H, 20}^{(4 s)}$ 


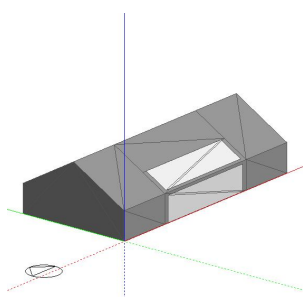

(a) 0\% Dir.Gains

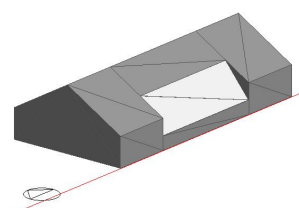

(d) $0 \%$ Dir.Gains

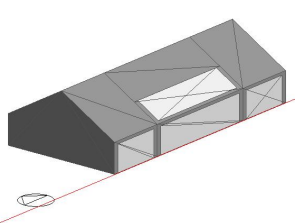

(b) $50 \%$ Dir.Gains

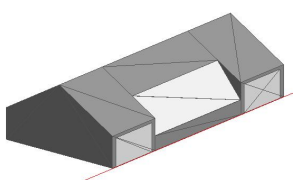
8

(e) $50 \%$ Dir.Gains

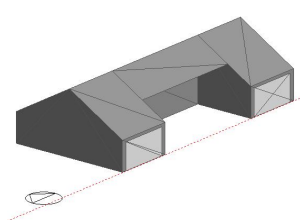

(c) $50 \%$ Dir.Gains

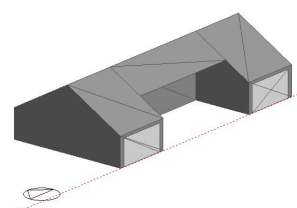

(f) $50 \%$ Dir.Gains

Figure 3: Integrated sunspace: B1 ( $a$ and b), B2 (d and e). No sunspace: B0 (c and f).

and $Q_{C, 20}^{(4 s)}$ for the sunspace. Using these results, the conditioned zone and sunspace heat transfer terms, both excluding the heat transferred through the sunspace partition wall, $Q_{h t}^{a d i a b}$ and $Q_{h t, s}$, respectively, are determined by

$$
\begin{gathered}
Q_{h t}^{a d i a b}=Q_{H, 20}^{(4)}-Q_{C, 20}^{(4)} \\
Q_{h t, s}=Q_{H, 20}^{(4 s)}-Q_{C, 20}^{(4 s)}
\end{gathered}
$$

In the aforementioned simulation the longwave extra radiation to the sky vault is also nulled by adjusting sky longwave radiation to the external air temperature, as described for the first building simulation. This parcel is, therefore, included in the heat source terms.

Furthermore, heat transfer between conditioned zone and external environment trough the sunspace partition wall $\left(Q_{h t, p}\right)$, could be obtained from

$$
Q_{h t, p}=Q_{h t}-Q_{h t}^{a d i a b}
$$

where $Q_{h t}$ is the general term calculated by equation (18).

Finally, $b_{t r}$ is calculated from

$$
b_{t r}=1-\frac{Q_{h t, p}}{Q_{h t, s}}
$$


One additional simulation is run for the sunspace zone, similar to simulation (4) but including solar gains, to get $Q_{H, 20}^{(5 s)}$ e $Q_{C, 20}^{(5 s)}$ which is used to compute

$$
\Phi_{u}=Q_{h t, s}-\left(Q_{H, 20}^{(5 s)}-Q_{C, 20}^{(5 s)}\right)
$$

\section{Simulation results}

\subsection{Internal gains}

Regarding the different approaches verified between ISO 13790 and SLR methods and in order to clarify the influence of internal gains on the heat transfer and heat sources terms, simulations are run for models A and B at different levels of internal gains 2 and $4 \mathrm{Wm}^{-2}$. Internal gains are constant during time and refer to the conditioned floor area. The method described in Section 3.2 is used to estimate, by simulation, the heat transfer and heat sources terms. $Q_{h t}^{(-)}$and $Q_{h t}^{(+)}$are the heat transfer calculated without and with internal gains, respectively, and no other gains (e.g. solar radiation) are considered in these simulations. In addition, $Q_{g n}^{(-)}$and $Q_{g n}^{(+)}$are the heat sources estimated by simulation for the same conditions; therefore the first term includes internal gains and the former does not. From the aforementioned terms the heat transfer-to-heat source ratio, $Q_{h t} / Q_{g n}$ (for the sake of simplicity, hereafter, loss-to-gain ratio) and the solar heating fraction are obtained. The sensitivity analysis shown in Fig. 4 does not take into account how indirect gains are estimated, because both solar heating fraction (SHF) and loss-to-gain ratio are directly obtained by simulation results.

From the results obtained, it can be concluded that, for all sunspaces types, there are slight differences between methodologies, assuming that internal gains are low compared to solar gains. Another interesting conclusion is that each type of sunspace, independently from the area of direct and indirect gains, is characterized by a single curve correlation expressed by equation (12). This is due to the way gains are computed, i.e. loss-to-gain ratio takes into account the effective gains entering in the conditioned zone, whereas they come from direct or indirect solar systems. Gains computation will be explored in the Section 4.3.

\subsection{Heat transfer through solar collector surfaces}

SLR methods, unlike ISO 13790, do not include the heat transfer through solar collectors surfaces, such as windows for direct gain and partition walls for sunspaces. The same simulations of Sec. 4.1 are used to study the influence of excluding heat transfer corresponding to solar collectors surfaces, here the windows and/or the partition wall between conditioned zone and 

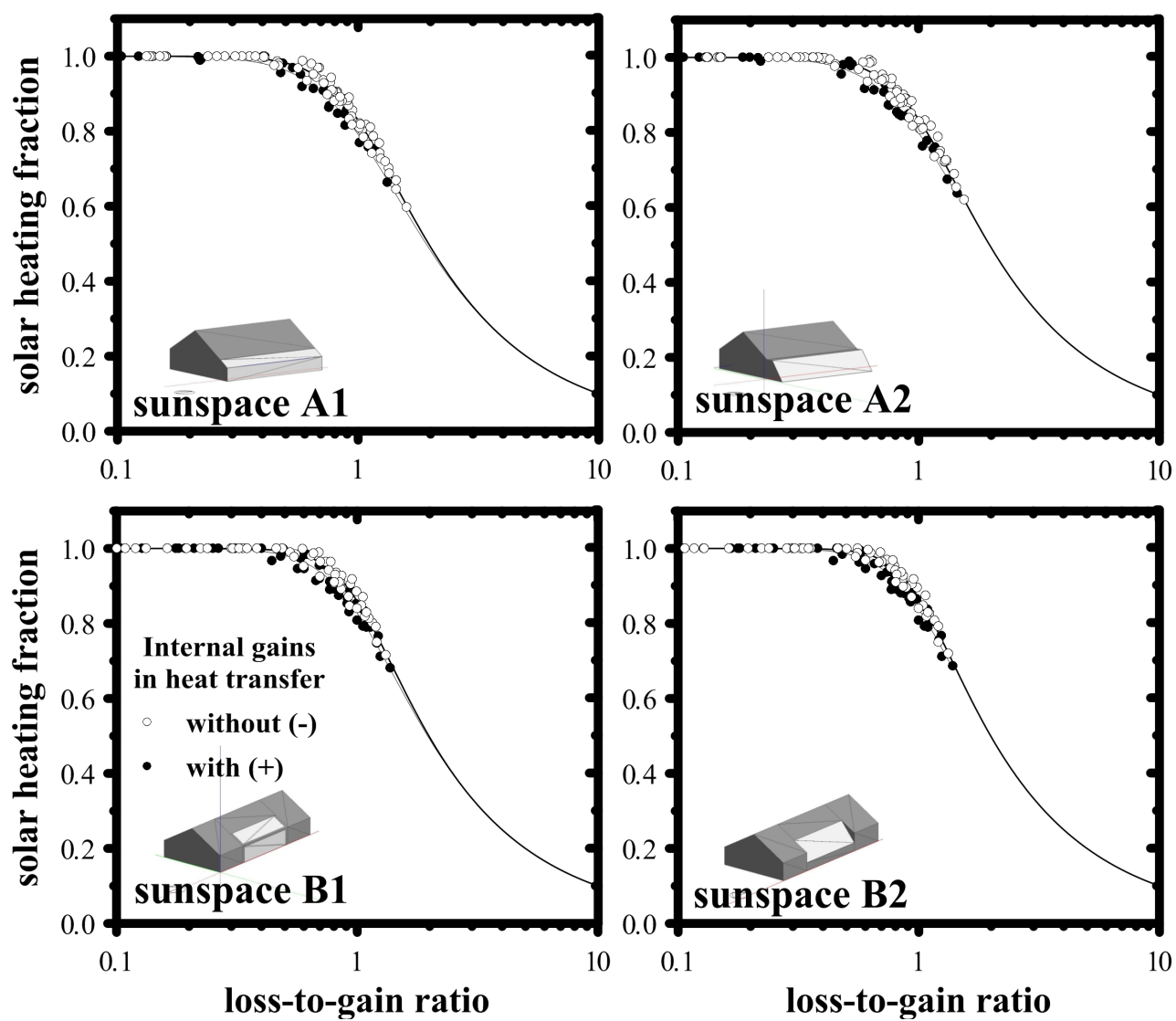

Figure 4: Monthly calculations where heat transfer is obtained without internal gains (-) and with internal gains $(+)$. X-axis logarithmic scale. Solid line is an empirical function, equation (17), fitted to the results. 
sunspace. The results are shown in Fig. 5 where dashed lines are the SLR empirical functions, equation (12), with the parameters of Table 4 for Balcomb and Jones [14]. As for internal gains sensitivity analysis, all terms heat transfer and heat gains - are directly obtained from simulation results.
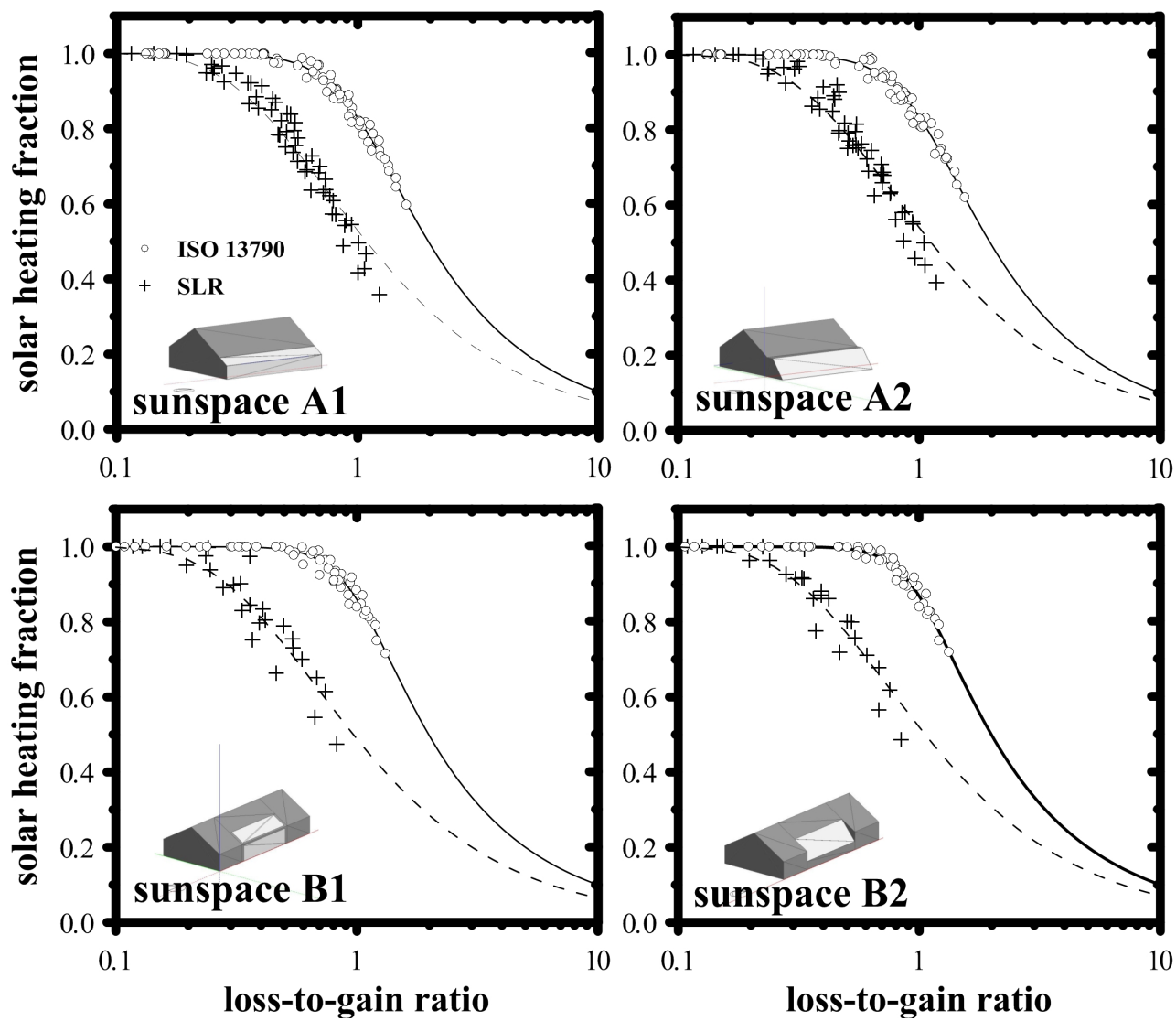

Figure 5: SLR and ISO 13790 monthly methods: solar collector surfaces and their inclusion in heat transfer term. X-axis logarithmic scale. Empirical functions fitted to the results: solid line uses equation (17) and dashed line uses equation (12).

An important conclusion is that the exclusion of $Q_{h t, p}$ (heat transfer through south windows and/or sunspace partition wall) is determinant for the correlation function: SLR correlation obtained by simulation results significantly differ from the obtained with ISO 13790 method, and approaches especially for B type - the empirical correlations of Balcomb and Jones [14], which parameters are also included in Table 4. 
Table 4: SLR parameters for sunspace types.

\begin{tabular}{lcccccc}
\hline & \multicolumn{3}{c}{ current models } & \multicolumn{3}{c}{ Balcomb and Jones $[14]$} \\
sunspace type & $b$ & $c$ & $\xi$ & $b$ & $c$ & $\xi$ \\
\hline A1 & 1 & 0.7525 & 0.70 & 0.9683 & 0.4954 & 0.84 \\
A2 & 1 & 0.7760 & 0.55 & 0.9587 & 0.4770 & 0.83 \\
B1 & 1 & 0.7334 & 0.15 & 0.9968 & 0.7004 & 0.77 \\
B2 & 1 & 0.6702 & 0.15 & 0.9889 & 0.6643 & 0.84
\end{tabular}

A attached, B integrated

\subsection{Indirect solar gains}

The study reported in the former two sections takes into account that indirect gains are obtained from simulation, which is the parcel of heat collected in the sunspace useful for the conditioned zone. But to apply ISO 13790 and SLR, the total amount of heat transferred to the conditioned zone by the sunspace $\left(Q_{s s}\right)$ should be estimated by the formulations shown in Table 2 .

The different formulations seek to determine which parcel of the heat collected in the sunspace should be considered as heat source (SLR and detailed method of ISO 13790) or subtracted to the heat transfer (simplified method of ISO 13790).

It is noteworthy that the subtraction of indirect gains from heat transfer in the simplified method of ISO 13790 is a similar procedure to that assumed for internal gains in SLR method. For simplicity reasons we assume that the heat generated inside the sunspace is equivalent to the heat absorbed by the sunspace surfaces, $\Phi_{u}=Q_{a b, s}$.

The comparison between methods (Fig. 6) shows that the detailed method of ISO 13790 is able to fairly reproduce the simulation results (solid line in Fig. 6). However, the simplified method of ISO 13790 fails to reproduce the correlations previously obtained with simulation results for:

- mid season months, where useful sunspace solar gains are slightly higher than reference heating load of conditioned zone corresponding to low loss-to-gain ratio, but computed as negative in the simplified method (not shown in Fig. 6);

- cold months, where losses are significantly higher than sunspace solar gains, high loss-to-gain ratio.

The calculation procedure of heat gains in SLR for sunspaces takes into account $\xi$, an adjustable empiric parameter obtained by curve fitting the results obtained by simulation. In this specific work, $\xi$ is obtained by linear 

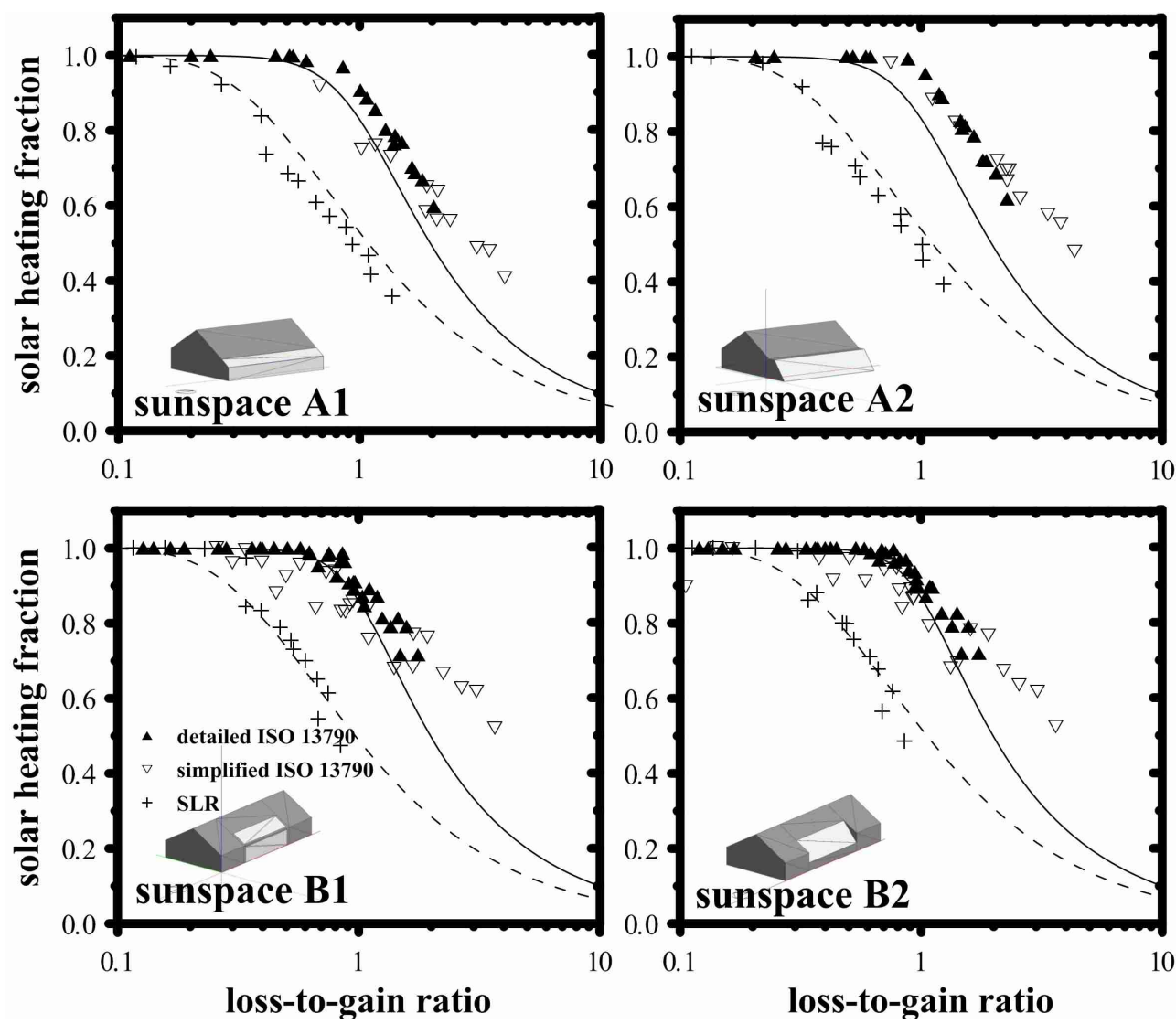

Figure 6: Monthly indirect gains quantification by method. Empirical functions fitted to the results: solid line uses equation (17) and dashed line uses equation (12). 
regression between the computed conditioned zone heat gains from the sunspace $\left(Q_{g n}\right.$ in equation (19) excluding internal gains) and the equation (11) numerator, for the coldest months. It is noteworthy that $Q_{h t, s}$ and $\Phi_{u}=Q_{a b, s}$ are also terms computed by equations (22) and (25), respectively. The obtained parameters (Table 4) are significantly lower than those proposed by Balcomb and Jones [14].

\subsection{Multiple solar systems}

In order to test the use of SLR to predict heating energy needs with combined direct and indirect solar gains, the results calculated according to equation (13) are compared with the simulations obtained in Fig. 7 for sunspaces A1 and A2, with $30 \%$ and $70 \%$ of projected area of direct gain.

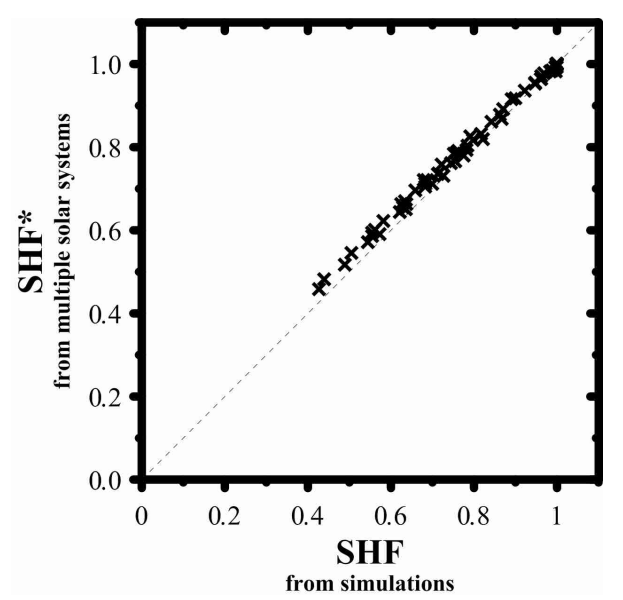

Figure 7: SLR method: combined direct and indirect gains.

It can be observed that the calculated $\mathrm{SHF}^{M}$ is biased from the simulation results by an average of 0.01 with a standard deviation corresponding to $\pm 1.7 \%$. This is in agreement with the results obtained by other authors [19] when applying the SLR method to a combination of solar systems.

\section{Integration of SLR correlations into ISO 13790}

\subsection{The new formulation}

When applying ISO 13790 to buildings with special elements, such as sunspaces, and given that SHF correlations are known, the explicit use of the loss utilization factor $\left(\eta_{l s}\right)$ in

$$
Q_{n d}=\left(1-\eta_{l s}\right) Q_{h t}
$$


has advantages when compared to the current formulation using the gain utilization factor, since $\eta_{l s}$ is equivalent to SHF. It is noteworthy that the use of SHF obtained by SLR assumes that heat transfer is not accounted for solar collector surfaces.

At this point, it is important to clarify that we are searching for a methodology that gives continuity to the formulation of ISO 13790, especially because there are some terms already accounted (e.g. direct gain). Therefore, $Q_{h t}$ should include the heat transfer through all elements that are boundaries for the conditioned zone, without exclusions.

Replacing SHF in equation (10) with (13) applied to a direct gain system $k$ and an indirect gain system $n$, results in

$$
Q_{n d}=\left(1-a_{p_{k}} \mathrm{SHF}_{k}-a_{p_{n}} \mathrm{SHF}_{n}\right) Q_{r e f}
$$

with $a_{p_{k}}$ and $a_{p_{n}}$ as the fraction projected area of the system $k$ and $n$, respectively.

In equation (27), the direct gain term is replaced by an equivalent formulation, which results from the assumption that, theoretically, heating energy needs are the same for ISO 13790 and SLR methodologies, so that

$$
\left(1-\mathrm{SHF}_{k}\right) Q_{r e f}=\left(1-\eta_{l s k}\right) Q_{h t}
$$

Rearranging (27) with (28), results in

$$
Q_{n d}=a_{p_{k}}\left(1-\eta_{l s k}\right) Q_{h t}+\left(1-a_{p_{k}}-a_{p_{n}} \mathrm{SHF}_{n}\right) Q_{r e f}
$$

which could also be generalized to include more than one indirect gain system

$$
Q_{n d}=a_{p_{k}}\left(1-\eta_{l s k}\right) Q_{h t}+\left[1-a_{p_{k}}-\sum_{n}\left(a_{p_{n}} \mathrm{SHF}_{n}\right)\right] Q_{r e f}
$$

This newly proposed formulation is compatible with ISO 13790 and applied to the same case studies of Section 4.4. Comparing the monthly heating energy needs with simulations (Fig. 8) it can be observed that the calculation method slightly underestimates the heating energy needs by an average of $12 M J$, with a standard deviation corresponding to $\pm 11.3 \%$.

\subsection{Final considerations}

It is noteworthy that the number of parameters needed to apply the detailed method in ISO 13790 for sunspaces does not differ significantly from SLR. However, the integration of SLR correlations in ISO 13790 can be useful when specific solar systems are already studied and defined in terms of known correlation functions. The importance of this study is in demonstrating that 


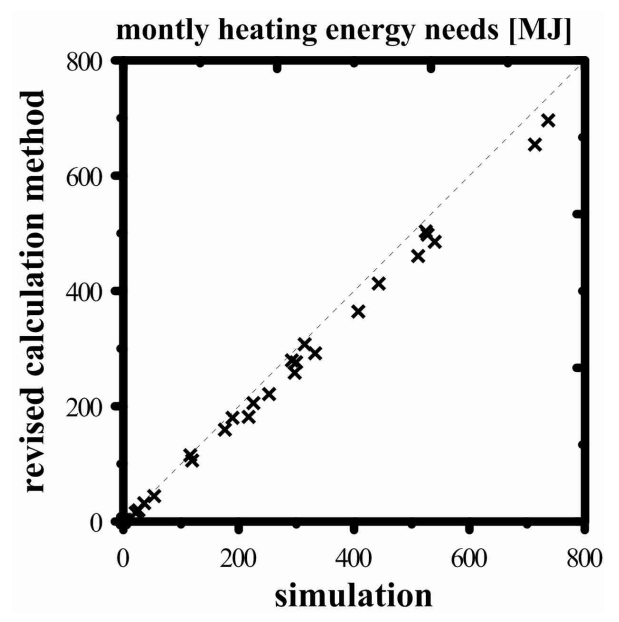

Figure 8: SLR integration in ISO 13790 compared to simulation.

methodologies can be integrated, even if they are not directly equivalent in terms of the parameters used by each method. Moreover, the application of the proposed methodology can be extended to other solar systems defined by SLR correlations, e.g. storage walls.

In synthesis, the sequence of procedures (see also Fig. 9) to apply equation (30), i.e. the general formulation for heating energy needs of multiple combined solar systems consists in:

- calculate the projected area - $a_{p}$ - of each solar system (direct and indirect gain);

- Applying ISO 13790 methodology, replace all projected area by direct gain (windows) and calculate heat transfer - $Q_{h t}$, heat sources - $Q_{g n}$, and the loss utilization factor $-\eta_{l s}$, ;

- Applying SLR methodology, for each indirect solar system, replace all the projected area of indirect gain solar system and calculate the reference heating load - $Q_{\text {ref }}$ (excluding solar collector surfaces), heat sources - $Q_{\text {sol }}$, and SLR parameter; for each indirect solar system calculate $\mathrm{SHF}$;

- Use equation 30 to calculate heating energy needs.

In terms of internal gains, SLR assumes that reference heating load, $Q_{r e f}$, is obtained with internal gains, corresponding to a decrease of the base temperature $T_{b}$, used for degree-days calculation, so that 


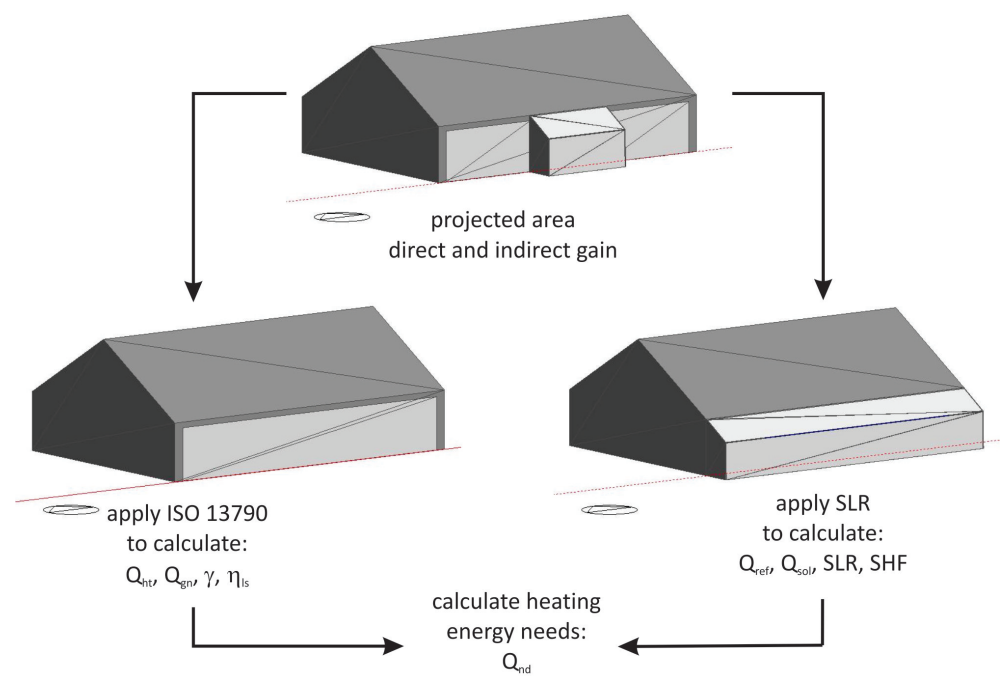

Figure 9: Schematic procedure for the integration of SLR method in ISO 13790.

$$
T_{b}=T_{r e f}-\frac{Q_{i}}{H_{t}-H_{p}}
$$

where $T_{r e f}$ is the reference value assumed for air temperature set point, $H_{t}$ the total conditioned zone conductance and $H_{p}$ the solar collector surfaces conductance.

\section{Conclusions}

The procedure established in standard ISO 13790 for indirect gains and Solar Load Ratio methodology are addressed in this work to access the possibility of integrating both methods.

The main contribution of the work presented here is the new formulation to account SLR (Solar Load Ratio) correlations in ISO 13790, here obtained for sunspaces, but generalizable for other solar systems with known SLR functions.

Using simulation models to perform a sensitivity analysis of different parameters leads to the observation that internal gains can be added to the heat source term (heat gains), as well as subtracted to the heat transfer term (heat losses) without influencing the utilization factor dependence with the gain-to-loss ratio. This approach distinguishes ISO 13790 from Load Ratio 
methods but it is not an obstacle to an integrative approach of both methods. This conclusion is valid for internal gains from typical residential use, i.e. low when compared with solar gains.

On the other hand, the SLR assumption that solar collector surfaces (windows, sunspace partition walls or storage walls) are neutral elements, which are not added to the heat transfer term results in large inconsistencies between SLR and ISO 13790 methods.

Moreover, the gain utilization factor it shown to be generally applied to all gains, including indirect gains, as assumed in ISO 13790. The most difficult issue is the quantification of solar gains, especially in the case of sunspaces with multiple transparent surfaces and the detailed methodology of ISO 13790 fairly reproduces the results obtained by simulation. Relatively to the simplified methodology of ISO 13790, where the heat generated inside the sunspace is accounted as a decrease of the adjustment factor applied to unoccupied spaces, it is shown that predictions fail in mid season months and coldest months.

\section{Nomenclature}

$A_{e}$ effective collection area $\left(m^{2}\right)$

$A_{p}$ glazing projected area $\left(m^{2}\right)$

$a$ building inertia constant

$a_{p}$ fraction of glazing projected area

$b$ adjustable empiric parameter

$b_{t r}$ sunspace adjustment factor

$b_{t r}^{*}$ corrected sunspace adjustment factor

$c$ adjustable empiric parameter

$H_{p}$ sunspace partition wall conductance $(W / K)$

$H_{s}$ sunspace external surfaces conductance $(W / K)$

$H_{t}$ total conditioned zone conductance $(W / K)$

$I$ surface monthly solar irradiation $\left(J / m^{2}\right)$

$Q_{a b, s}$ solar radiation absorbed by the sunspace massive surfaces $(J)$ 
$Q_{C, 20}$ cooling energy needs for air temperature set point equal to $20^{\circ} \mathrm{C}(\mathrm{J})$

$Q_{g n}$ heat sources $(J)$

$Q_{H, 20}$ heating energy needs for air temperature set point equal to $20^{\circ} \mathrm{C}(\mathrm{J})$

$Q_{h t, p}$ heat transfer through solar colector surfaces $(J)$

$Q_{h t, p}^{*}$ corrected heat transfer through solar colector surfaces $(J)$

$Q_{h t, s}$ sunspace heat transfer $(J)$

$Q_{h t}$ heat transfer $(J)$

$Q_{h t}^{a d i a b}$ heat transfer considering an adiabatic solar colector surface $(J)$

$Q_{i}$ conditioned zone internal gains $(J)$

$Q_{n d}$ heating energy needs $(J)$

$Q_{\text {ref }}$ reference heating load $(J)$

$Q_{s d, p}$ conditioned zone direct gains from the opaque surfaces of sunspace partition wall $(J)$

$Q_{s d, t}$ conditioned zone direct gains from the transparent surfaces of sunspace partition wall $(J)$

$Q_{s d, w}$ conditioned zone direct gains from windows $(J)$

$Q_{s i}$ conditioned zone indirect gains from sunspace $(J)$

$Q_{\text {sol }}$ collected solar gains $(J)$

$Q_{s s}$ total heat going from the sunspace to the conditioned zone $(J)$

SHF Solar Heating Fraction

SHF $^{M}$ equivalent SHF for multiple solar systems

SLR Solar Load Ratio

SLR $^{*}$ corrected Solar Load Ratio

$T_{b}$ base temperature for degree-days calculation $(K)$

$T_{\text {ext }}$ monthly average of external air temperature $(K)$ 
$T_{\text {ref }}$ reference value assumed for air temperature set point $(K)$

Greek letters

$\alpha$ surface solar absorption coefficient

$\eta_{g n}$ gain utilization factor

$\eta_{l s}$ loss utilization factor

$\gamma$ heat sources-to-heat transfer ratio

$\phi$ non utilizability zero thermal inertia

$\Phi_{u}$ heat generated within an unconditioned zone $(J)$

$\tau$ building time constant $(h)$

$\xi$ adjustable empiric parameter

Subscripts

i solar system

j surface

$\mathbf{k}$ direct gain solar system

$\mathbf{n}$ indirect gain solar system

Superscripts
(1) first simulation
(2) second simulation
(3) third simulation
(4) forth simulation

(4s) forth simulation applied to sunspace

(5s) fifth simulation applied to sunspace

(-) without internal gains in heat transfer

$(+)$ with internal gains in heat transfer 


\section{References}

[1] CEC. Energy Performance of Building Directive, Directive 2010/31/EU. Official Journal of the European Communities; 2010:13-35.

[2] Marszal AJ, Heiselberg P, Bourrelle JS, Musall E, Voss K, Sartori I, Napolitano A. Zero Energy Building: A review of definitions and calculation methodologies. Energy and Buildings 2011;43,971979.

[3] CEC. Energy Performance of Building Directive, Directive 2002/91/EC. Official Journal of the European Communities; 2003:65-71.

[4] ISO 13790 Energy performance of buildings, calculation of energy use for space heating and cooling; 2008.

[5] Balcomb JD, McFarland RD. A simple empirical method for estimating the performance of passive solar heated building of the thermal storage wall type, in Proc. 2th National Passive Solar Conferences, ISES (USA), 1978.

[6] Mazria E. The Passive Solar Energy Book: A Complete Guide to Passive Solar Home, Greenhouse and Building Design. Emmaus: Rodale Press; 1979 .

[7] Monsen WA, Klein SA, Beckman WA. The Un-Utilizability Design for Collector Storage Walls. Solar Energy 1982;29,421-429.

[8] Sander DM, Barakat SA. A method for estimating the utilization of solar gains trhough windows. ASHRAE Transactions 1993;89,1A,12-22.

[9] Mihalakakou G, Ferrante A. Energy conservation and potential of a sunspace: sensitivity analysis. Energy Conservation Management $2004 ; 41,1247-1264$.

[10] Mihalakakou G. On the use of sunspace for space heating/cooling in Europe. Renewable Energy 2002;26,415-429.

[11] van Dijk D, Spiekman M. Energy performance of buildings; outline for harmonised EP procedures. Final report of EU SAVE ENPER project, Task B6. Delft (NL): TNO Building and Construction Research; June $29,2004$.

[12] ISO 13789 Thermal performance of buildings Transmission and ventilation heat transfer coefficients, calculation method; 2007. 
[13] Wray WO, Balcomb JD, McFarland RD. A semi-empirical method for estimating the performance of direct gain passive solar heated buildings, in Proc. 3th National Passive Solar Conferences, ISES (USA), 1979.

[14] Balcomb JD, Jones RW. Passive Solar Design Handbook, Passive Solar Design Analysis and Supplement, Vol III, American Solar Energy Society Inc., Boulder, New York, 1982.

[15] Oliveira AC, Oliveira Fernandes E. A new simplified method for evaluating the thermal behaviour of direct gain passive solar buildings. Solar Energy 1992,48;227-233.

[16] Corrado V, Fabrizio E. Assessment of building cooling energy need through a quasi-steady state model: simplified correlation for gain-loss mismatch. Energy and Buildings 2007;39:569-579.

[17] Oliveira Panão MJN, Camelo SML and Gonąlves HJP. Assessment of the Portuguese building thermal code: new revised requirements for cooling energy needs to prevent buildings summer overheating. Energy 2011,36;3262-3271.

[18] EnergyPlus Development Team. Input output reference: the encyclopedic reference to EnergyPlus input and output. EnergyPlus, 2010, Version 6.0 .

[19] Goncalves HJP. Interacção de sistemas solares passivos de ganho directo e indirecto, estudo numérico e experimental. PhD thesis, Porto; 1990. 